\title{
Article
}

\section{CERTAIN INEQUALITIES FOR THE CONFORMABLE GAMMA AND POLYGAMMA FUNCTIONS}

\author{
KWARA NANTOMAH
}

\begin{abstract}
In a recent paper, Sarikaya et al. introduced a new analogue of the classical Gamma function which they called, the conformable Gamma function. Motivated by their results, this paper seeks to establish some inequalities for the conformable Gamma and Polygamma functions. Among other analytical tools, the procedure relies on the generalized forms of some classical inequalities.
\end{abstract}

\section{Introduction and Preliminaries}

The classical Euler's Gamma function, which is an extension of the factorial notation to noninteger values may be defined for $x>0$ by any of the following equivalent forms.

$$
\begin{aligned}
\Gamma(x) & =\int_{0}^{\infty} t^{x-1} e^{-t} d t \\
& =\lim _{n \rightarrow \infty} \frac{n ! n^{x}}{x(x+1)(x+2) \ldots(x+n)} \\
& =\frac{1}{x e^{\gamma x}} \prod_{n=1}^{\infty} e^{\frac{x}{n}}\left(1+\frac{x}{n}\right)^{-1}
\end{aligned}
$$

where $\gamma=\lim _{n \rightarrow \infty}\left(\sum_{k=1}^{n} \frac{1}{k}-\ln n\right)=0.577215664 \ldots$ is the Euler-Mascheroni's constant. It is well known that the Gamma function satisfies the basic relation:

$$
\begin{aligned}
& \Gamma(x+1)=x \Gamma(x), \quad x \in \mathbb{R}^{+} \\
& \Gamma(n+1)=n !, \quad n \in \mathbb{Z}^{+} \cup\{0\}
\end{aligned}
$$

Closely related to the Gamma function are the Digamma and Polygamma functions which are respectively defined as

$$
\psi(x)=\frac{d}{d x} \ln \Gamma(x)=-\gamma-\frac{1}{x}+\sum_{n=1}^{\infty} \frac{x}{n(n+x)}
$$

and

$$
\psi^{(m)}(x)=\frac{d^{m}}{d x^{m}} \psi(x)=(-1)^{m+1} m ! \sum_{n=0}^{\infty} \frac{1}{(n+x)^{m+1}}, \quad m \in \mathbb{N}_{0}
$$

where $\psi^{(0)}(x) \equiv \psi(x)$.

2010 Mathematics Subject Classification. 33B15, 26D15.

Key words and phrases. Gamma function; conformable Gamma function; inequality. 
Euler gave another definition of the Gamma function which is called the $p$ analogue or $p$-Gamma function. This is defined as

$$
\Gamma_{p}(x)=\frac{p ! p^{x}}{x(x+1) \ldots(x+p)}=\frac{p^{x}}{x\left(1+\frac{x}{1}\right) \ldots\left(1+\frac{x}{p}\right)}
$$

where $\lim _{p \rightarrow \infty} \Gamma_{p}(x)=\Gamma(x)$. Since then, several analogues (or generalizations) of the Gamma function have been introduced and investigated. See for instance [5], [3], [4], [6], [7], [9] and [8] for the various analogues. Then in a recent paper [10], the authors introduce another version of the Gamma function called the conformable Gamma function defined as follows.

Let $\Gamma_{\alpha, k}(x)=\Gamma_{k}^{\alpha}(x)$ be the conformable Gamma function or the $(\alpha, k)$-analogue of the Gamma function introduced by Sarikaya et al. in [10]. Then $\Gamma_{\alpha, k}(x)$ is defined for $k>0, \alpha \in(0,1]$ and $x>0$ as

$$
\begin{aligned}
\Gamma_{\alpha, k}(x) & =\int_{0}^{\infty} t^{x-1} e^{-\frac{t^{\alpha k}}{\alpha k}} d_{\alpha} t \\
& =\lim _{n \rightarrow \infty} \frac{n ! \alpha^{n} k^{n}(n \alpha k)^{\frac{x+\alpha-1}{\alpha k}-1}}{(x)_{n, k}^{\alpha}}
\end{aligned}
$$

where

$$
(x)_{n, k}^{\alpha}=(x+\alpha-1)(x+\alpha-1+\alpha k)(x+\alpha-1+2 \alpha k) \cdots(x+\alpha-1+(n-1) \alpha k)
$$

is the $(\alpha, k)$-Pochhammer symbol. Clearly, $\Gamma_{1,1}(x)=\Gamma(x)$. In addition, it has been shown in [10] that the function $\Gamma_{\alpha, k}(x)$ satisfies the following identities.

$$
\begin{aligned}
\Gamma_{\alpha, k}(x+\alpha k) & =(x+\alpha-1) \Gamma_{\alpha, k}(x) \\
\Gamma_{\alpha, k}(x) & =(\alpha k)^{\frac{x+\alpha-1}{\alpha k}-1} \Gamma\left(\frac{x+\alpha-1}{\alpha k}\right) \\
\Gamma_{\alpha, k}(x) & =(\alpha)^{\frac{x+\alpha-1}{\alpha k}-1} \Gamma_{k}\left(\frac{x+\alpha-1}{\alpha}\right) \\
\Gamma_{\alpha, k}(\alpha k+1-\alpha) & =1
\end{aligned}
$$

where $\Gamma_{k}(x)$ is the $k$-analogue of the Gamma function.

This paper seeks to establish some inequalities for the conformable Gamma and Polygamma functions. We begin by recalling the following lemmas which shall be required in order to prove our results.

\section{Some Lemmas}

Lemma 2.1 (Generalized Hölder's Inequality for Integrals). Let $f_{1}, f_{2}, \ldots, f_{n}$ be functions such that the integrals exist. Then the inequality

$$
\int_{a}^{b}\left|\prod_{i=1}^{n} f_{i}(t)\right| d t \leq \prod_{i=1}^{n}\left(\int_{a}^{b}\left|f_{i}(t)\right|^{\lambda_{i}} d t\right)^{\frac{1}{\lambda_{i}}}
$$

holds for $\lambda_{i}>1$ such that $\sum_{i=1}^{n} \frac{1}{\lambda_{i}}=1$. 
Lemma 2.2 (Generalized Minkowski's Inequality for Integrals). Let $f_{1}, f_{2}, \ldots, f_{n}$ be functions such that the integrals exist. Then the inequality

$$
\left(\int_{a}^{b}\left|\sum_{i=1}^{n} f_{i}(t)\right|^{u} d t\right)^{\frac{1}{u}} \leq \sum_{i=1}^{n}\left(\int_{a}^{b}\left|f_{i}(t)\right|^{u} d t\right)^{\frac{1}{u}}
$$

holds for $u \geq 1$.

Lemma 2.3 (Generalized Hölder's Inequality for Sums). Let $i=1,2,3, \ldots, N$ and $n=1,2,3, \ldots, T$ such that the sums exist. Then the inequality

$$
\sum_{n=1}^{T}\left|\prod_{i=1}^{N} Q_{i, n}\right| \leq \prod_{i=1}^{N}\left(\sum_{n=1}^{T}\left|Q_{i, n}\right|^{\lambda_{i}}\right)^{\frac{1}{\lambda_{i}}}
$$

is valid for $\lambda_{i}>1$ such that $\sum_{i=1}^{N} \frac{1}{\lambda_{i}}=1$.

Lemma 2.4 (Generalized Minkowski's Inequality for Sums). Let $i=1,2,3, \ldots, N$ and $n=1,2,3, \ldots, T$ such that the sums exist. Then the inequality

$$
\left(\sum_{n=1}^{T}\left|\sum_{i=1}^{N} Q_{i, n}\right|^{u}\right)^{\frac{1}{u}} \leq \sum_{i=1}^{N}\left(\sum_{n=1}^{T}\left|Q_{i, n}\right|^{u}\right)^{\frac{1}{u}}
$$

is valid for $u \geq 1$.

Lemma 2.5 (Weighted AM-GM Inequality). For $i=1,2, \ldots, n$, let $Q_{i} \geq 0$ and $\lambda_{i}>1$ such that $\sum_{i=1}^{n} \frac{1}{\lambda_{i}}=1$. Then the inequality

$$
\sum_{i=1}^{n} \frac{1}{\lambda_{i}} Q_{i} \geq \prod_{i=1}^{n} Q_{i}^{\frac{1}{\lambda_{i}}}
$$

holds.

Remark 2.6. Lemma 2.5 is well-known in the literature, and the proofs Lemmas $2.1,2.2,2.3$ and 2.4 can be found in pages $790-791$ of [2].

\section{Inequalities for the Conformable Gamma Function}

This section is devoted to some inequalities involving the conformable Gamma function, $\Gamma_{\alpha, k}(x)$.

Proposition 3.1. The function $\Gamma_{\alpha, k}(x)$ satisfies the infinite product representation:

$$
\Gamma_{\alpha, k}(x)=\frac{(\alpha k)^{\frac{x+\alpha-1}{\alpha k}}}{x+\alpha-1} e^{-\gamma\left(\frac{x+\alpha-1}{\alpha k}\right)} \prod_{n=1}^{\infty}\left[\left(1+\frac{x+\alpha-1}{\alpha n k}\right)^{-1} e^{\frac{x+\alpha-1}{\alpha n k}}\right]
$$

Proof. This follows directly from (11) and (3).

Let $\psi_{\alpha, k}(x)$ be the conformable Digamma function defined as the logarithmic derivative of $\Gamma_{\alpha, k}(x)$. By direct computations using (18), we obtain 


$$
\begin{aligned}
\psi_{\alpha, k}(x) & =\frac{d}{d x} \ln \Gamma_{\alpha, k}(x) \\
& =\frac{\ln (\alpha k)-\gamma}{\alpha k}-\frac{1}{x+\alpha-1}+\sum_{n=1}^{\infty}\left[\frac{1}{n \alpha k}-\frac{1}{n \alpha k+x+\alpha-1}\right]
\end{aligned}
$$

Also, let $\psi_{\alpha, k}^{(m)}(x)$ be the conformable Polygamma function defined as the $m$-th derivative of $\psi_{\alpha, k}(x)$. That is,

$$
\psi_{\alpha, k}^{(m)}(x)=\frac{d^{m}}{d x^{m}} \psi_{\alpha, k}(x)=\sum_{n=0}^{\infty} \frac{(-1)^{m+1} m !}{(n \alpha k+x+\alpha-1)^{m+1}}
$$

where $m \in \mathbb{N}_{0}$ and $\psi_{\alpha, k}^{(0)} \equiv \psi_{\alpha, k}(x)$.

Proposition 3.2. The following statements are valid.

(a) $\psi_{\alpha, k}(x)$ is increasing on $(0, \infty)$.

(b) $\psi_{\alpha, k}^{(m)}(x)$ is positive and decreasing on $(0, \infty)$ if $m$ is odd.

(c) $\psi_{\alpha, k}^{(m)}(x)$ is negative and increasing on $(0, \infty)$ if $m$ is even.

Proof. These follow directly from (20).

Theorem 3.3. Let $\lambda_{i}>1, i=1,2, \ldots, n$ and $\sum_{i=1}^{n} \frac{1}{\lambda_{i}}=1$. Then the inequality

$$
\Gamma_{\alpha, k}\left(\sum_{i=1}^{n} \frac{x_{i}}{\lambda_{i}}\right) \leq \prod_{i=1}^{n}\left(\Gamma_{\alpha, k}\left(x_{i}\right)\right)^{\frac{1}{\lambda_{i}}}
$$

is holds for $x_{i}>0$.

Proof. By (8) and (13), we obtain

$$
\begin{aligned}
\Gamma_{\alpha, k}\left(\sum_{i=1}^{n} \frac{x_{i}}{\lambda_{i}}\right) & =\int_{0}^{\infty} t^{\sum_{i=1}^{n} \frac{x_{i}}{\lambda_{i}}-1} e^{-\frac{t^{\alpha k}}{\alpha k}} d_{\alpha} t \\
& =\int_{0}^{\infty} t^{\sum_{i=1}^{n} \frac{x_{i}-1}{\lambda_{i}}} e^{-\frac{t^{\alpha k}}{\alpha k} \cdot \sum_{i=1}^{n} \frac{1}{\lambda_{i}}} d_{\alpha} t \\
& =\int_{0}^{\infty} \prod_{i=1}^{n}\left(t^{\frac{x_{i}-1}{\lambda_{i}}} e^{-\frac{t^{\alpha k}}{\alpha k} \cdot \frac{1}{\lambda_{i}}}\right) d_{\alpha} t \\
& \leq \prod_{i=1}^{n}\left(\int_{0}^{\infty} t^{x_{i}-1} e^{-\frac{t^{\alpha k}}{\alpha k}} d_{\alpha} t\right)^{\frac{1}{\lambda_{i}}} \\
& =\prod_{i=1}^{n}\left(\Gamma_{\alpha, k}\left(x_{i}\right)\right)^{\frac{1}{\lambda_{i}}}
\end{aligned}
$$

which completes the proof.

Remark 3.4. In particular, by letting $n=2, \lambda_{1}=p, \lambda_{2}=q, x_{1}=x$ and $x_{2}=y$ in (21), we obtain

$$
\Gamma_{\alpha, k}\left(\frac{x}{p}+\frac{y}{q}\right) \leq\left(\Gamma_{\alpha, k}(x)\right)^{\frac{1}{p}}\left(\Gamma_{\alpha, k}(y)\right)^{\frac{1}{q}}
$$


which is the result of Theorem 4.4 of [1].

Remark 3.5. Inequality (22) is another way of saying that the function $\Gamma_{\alpha, k}(x)$ is logarithmically convex. This fact can also be established by noting from (19) that $\frac{d^{2}}{d x^{2}} \ln \Gamma_{\alpha, k}(x)=\psi_{\alpha, k}^{\prime}(x)=\sum_{n=0}^{\infty} \frac{1}{(n \alpha k+x+\alpha-1)^{2}}>0$.

Remark 3.6. Furthermore, if $n=2, \lambda_{1}=\lambda_{2}=2, x_{1}=x$ and $x_{2}=y$ in (21), then we obtain the result

$$
\Gamma_{\alpha, k}\left(\frac{x+y}{2}\right) \leq \sqrt{\Gamma_{\alpha, k}(x) \Gamma_{\alpha, k}(y)} .
$$

Theorem 3.7. Let $s \geq 1$. Then the inequality

$$
\Gamma_{\alpha, k}(s x) \leq(\alpha k)^{\frac{x}{\alpha k}(s-1)} \Gamma_{\alpha, k}(x)
$$

holds if $0<x \leq 1$ and it reverses if $x \geq 2$.

Proof. By (11), we have

$$
\frac{\Gamma_{\alpha, k}(s x)}{\Gamma_{\alpha, k}(x)} \leq(\alpha k)^{\frac{x}{\alpha k}(s-1)} \frac{\Gamma\left(\frac{s x+\alpha-1}{\alpha k}\right)}{\Gamma\left(\frac{x+\alpha-1}{\alpha k}\right)} .
$$

Then (24) follows easily from this since $\Gamma(x)$ is decreasing on $(0,1]$.

Corollary 3.8. The inequality

$$
\Gamma_{\alpha, k}(x+y) \leq(\alpha k)^{\frac{x+y}{2 \alpha k}} \sqrt{\Gamma_{\alpha, k}(x) \Gamma_{\alpha, k}(y)} .
$$

holds for $0<x \leq 1$.

Proof. By using (23) and (24) for $s=2$, we obtain

$$
\begin{aligned}
\Gamma_{\alpha, k}(x+y) & \leq \sqrt{\Gamma_{\alpha, k}(2 x) \Gamma_{\alpha, k}(2 y)} \\
& \leq(\alpha k)^{\frac{x+y}{2 \alpha k}} \sqrt{\Gamma_{\alpha, k}(x) \Gamma_{\alpha, k}(y)}
\end{aligned}
$$

as desired.

Theorem 3.9. The inequality

$$
\begin{aligned}
\exp \left(\frac{1-u}{2}\left(\psi_{\alpha, k}(x+u)+\psi_{\alpha, k}(x+1)\right)\right) & \leq \frac{\Gamma_{\alpha, k}(x+1)}{\Gamma_{\alpha, k}(x+u)} \leq \\
& \exp \left((1-u) \psi_{\alpha, k}\left(x+\frac{1+u}{2}\right)\right)
\end{aligned}
$$

is holds for $0 \leq u \leq 1$ and $x>0$.

Proof. We make use of the Hermite-Hadamard's inequality which states that: if $f(x)$ is convex on $[a, b]$, then

$$
f\left(\frac{a+b}{2}\right) \leq \frac{1}{b-a} \int_{a}^{b} f(x) d x \leq \frac{f(a)+f(b)}{2} .
$$


Let $f(x)=-\psi_{\alpha, k}(x), a=x+u$ and $b=x+1$. Then $f(x)$ is convex and by (27) we obtain

$$
-\psi_{\alpha, k}\left(x+\frac{u+1}{2}\right) \leq-\frac{1}{1-u} \int_{x+u}^{x+1} \psi_{\alpha, k}(t) d t \leq-\frac{\psi_{\alpha, k}(x+u)+\psi_{\alpha, k}(x+1)}{2}
$$

which implies

$$
\frac{\psi_{\alpha, k}(x+u)+\psi_{\alpha, k}(x+1)}{2} \leq \frac{1}{1-u} \ln \frac{\Gamma_{\alpha, k}(x+1)}{\Gamma_{\alpha, k}(x+u)} \leq \psi_{\alpha, k}\left(x+\frac{u+1}{2}\right) .
$$

Then by taking exponents, we obtain the result (26).

Theorem 3.10. The inequality

$$
\left(\sum_{i=1}^{n} \Gamma_{\alpha, k}\left(x_{i}\right)\right)^{\frac{1}{u}} \leq \sum_{i=1}^{n}\left(\Gamma_{\alpha, k}\left(x_{i}\right)\right)^{\frac{1}{u}}
$$

holds for $x_{i}>0, i=1,2, \ldots, n$ and $u \geq 1$.

Proof. By (8) we obtain

$$
\begin{aligned}
\left(\sum_{i=1}^{n} \Gamma_{\alpha, k}\left(x_{i}\right)\right)^{\frac{1}{u}} & =\left(\sum_{i=1}^{n} \int_{0}^{\infty} t^{x_{i}-1} e^{-\frac{t^{\alpha k}}{\alpha k}} d_{\alpha} t\right)^{\frac{1}{u}} \\
& =\left(\int_{0}^{\infty}\left[\sum_{i=1}^{n}\left(t^{\frac{x_{i}-1}{u}} e^{-\frac{t^{\alpha k}}{\alpha k u}}\right)^{u}\right]_{\alpha} t\right)^{\frac{1}{u}} \\
& \leq\left(\int_{0}^{\infty}\left[\sum_{i=1}^{n}\left(t^{\frac{x_{i}-1}{u}} e^{-\frac{t^{\alpha k}}{\alpha k u}}\right)^{u} d_{\alpha} t\right)^{\frac{1}{u}}\right. \\
& \leq \sum_{i=1}^{n}\left(\int_{0}^{\infty}\left(t^{\frac{x_{i}-1}{u}} e^{-\frac{t^{\alpha k}}{\alpha k u}}\right)^{u} d_{\alpha} t\right)^{\frac{1}{u}} \\
& =\sum_{i=1}^{n}\left(\int_{0}^{\infty} t^{x_{i}-1} e^{-\frac{t^{\alpha k}}{\alpha k}} d_{\alpha} t\right)^{\frac{1}{u}} \\
& =\sum_{i=1}^{n}\left(\Gamma_{\alpha, k}\left(x_{i}\right)\right)^{\frac{1}{u}}
\end{aligned}
$$

which completes the proof. Note: The first inequality in this proof follows from the fact that $\sum_{i=1}^{n} a_{i}^{u} \leq\left(\sum_{i=1}^{n} a_{i}\right)^{u}$ for $a_{i} \geq 0, u \geq 1$ while the second inequality is as a result of the generalized Minkowski's inequality (14).

Remark 3.11. Let $n=2, x_{1}=x$ and $x_{2}=y$ in (28). Then we obtain

$$
\left(\Gamma_{\alpha, k}(x)+\Gamma_{\alpha, k}(y)\right)^{\frac{1}{u}} \leq\left(\Gamma_{\alpha, k}(x)\right)^{\frac{1}{u}}+\left(\Gamma_{\alpha, k}(y)\right)^{\frac{1}{u}} .
$$

Theorem 3.12. The inequality

$$
\exp \Gamma_{\alpha, k}\left(\sum_{i=1}^{n} \frac{x_{i}}{\lambda_{i}}\right) \leq \prod_{i=1}^{n}\left(\exp \Gamma_{\alpha, k}\left(x_{i}\right)\right)^{\frac{1}{\lambda_{i}}}
$$


holds for $x_{i}>0, i=1,2, \ldots, n, \lambda_{i}>1$ and $\sum_{i=1}^{n} \frac{1}{\lambda_{i}}=1$.

Proof. By (8) and the Weighted AM-GM inequality (17), we obtain

$$
\begin{aligned}
& \Gamma_{\alpha, k}\left(\sum_{i=1}^{n} \frac{x_{i}}{\lambda_{i}}\right)-\sum_{i=1}^{n} \frac{\Gamma_{\alpha, k}\left(x_{i}\right)}{\lambda_{i}} \\
& =\int_{0}^{\infty} t^{\sum_{i=1}^{n} \frac{x_{i}}{\lambda_{i}}-1} e^{-\frac{t^{\alpha k}}{\alpha k}} d_{\alpha} t-\sum_{i=1}^{n} \frac{1}{\lambda_{i}} \int_{0}^{\infty} t^{x_{i}-1} e^{-\frac{t^{\alpha k}}{\alpha k}} d_{\alpha} t \\
& =\int_{0}^{\infty} \prod_{i=1}^{n} t^{\frac{x_{i}-1}{\lambda_{i}}} e^{-\frac{t^{\alpha k}}{\alpha k}} d_{\alpha} t-\int_{0}^{\infty} \sum_{i=1}^{n} \frac{1}{\lambda_{i}} t^{x_{i}-1} e^{-\frac{t^{\alpha k}}{\alpha k}} d_{\alpha} t \\
& =\int_{0}^{\infty}\left[\prod_{i=1}^{n} t^{\frac{x_{i}-1}{\lambda_{i}}}-\sum_{i=1}^{n} \frac{1}{\lambda_{i}} t^{x_{i}-1}\right] e^{-\frac{t^{\alpha k}}{\alpha k}} d_{\alpha} t \\
& \leq 0 .
\end{aligned}
$$

That is,

$$
\Gamma_{\alpha, k}\left(\sum_{i=1}^{n} \frac{x_{i}}{\lambda_{i}}\right) \leq \sum_{i=1}^{n} \frac{\Gamma_{\alpha, k}\left(x_{i}\right)}{\lambda_{i}} .
$$

Then by exponentiating (30), we obtain the desired result (29).

\section{Inequalities for the Conformable Polygamma Function}

In this section, we establish some inequalities for the conformable Polygamma function $\psi_{\alpha, k}^{(m)}(x)$.

Theorem 4.1. For $i=1,2, \ldots, s$, let $\lambda_{i}>1, \sum_{i=1}^{s} \frac{1}{\lambda_{i}}=1, m_{i} \in \mathbb{N}$ and $\sum_{i=1}^{s} \frac{m_{i}}{\lambda_{i}} \in \mathbb{N}$. Then the inequality

$$
\left|\psi_{\alpha, k}^{\left(\sum_{i=1}^{s} \frac{m_{i}}{\lambda_{i}}\right)}\left(\sum_{i=1}^{s} \frac{x_{i}}{\lambda_{i}}\right)\right| \leq \prod_{i=1}^{s}\left|\psi_{\alpha, k}^{\left(m_{i}\right)}\left(x_{i}\right)\right|^{\frac{1}{\lambda_{i}}}
$$

holds for $x_{i}>0$.

Proof. By letting $\alpha=k=1$ in (21), we obtain $\Gamma\left(\sum_{i=1}^{n} \frac{x_{i}}{\lambda_{i}}\right) \leq \prod_{i=1}^{n}\left(\Gamma\left(x_{i}\right)\right)^{\frac{1}{\lambda_{i}}}$ and since the factorial is a special of the Gamma function, it follows that $\left(\sum_{i=1}^{n} \frac{m_{i}}{\lambda_{i}}\right) ! \leq$ 
$\prod_{i=1}^{n}\left(m_{i} !\right)^{\frac{1}{\lambda_{i}}}$. Then by using $(20)$ and $(15)$ we obtain

$$
\begin{aligned}
& \left|\psi_{\alpha, k}^{\left(\sum_{i=1}^{s} \frac{m_{i}}{\lambda_{i}}\right)}\left(\sum_{i=1}^{s} x_{i}\right)\right|=\sum_{n=0}^{\infty} \frac{\left(\sum_{i=1}^{s} \frac{m_{i}}{\lambda_{i}}\right) !}{\left(n \alpha k+\sum_{i=1}^{s} x_{i}+\alpha-1\right)^{\sum_{i=1}^{s} \frac{m_{i}}{\lambda_{i}}+1}} \\
& \leq \sum_{n=0}^{\infty} \frac{\prod_{i=1}^{s}\left(m_{i} !\right)^{\frac{1}{\lambda_{i}}}}{\left(n \alpha k+\sum_{i=1}^{s} x_{i}+\alpha-1\right)^{\sum_{i=1}^{s} \frac{m_{i}+1}{\lambda_{i}}}} \\
& \leq \sum_{n=0}^{\infty} \frac{\prod_{i=1}^{s}\left(m_{i} !\right)^{\frac{1}{\lambda_{i}}}}{\left(n \alpha k+x_{i}+\alpha-1\right)^{\sum_{i=1}^{s} \frac{m_{i}+1}{\lambda_{i}}}} \\
& =\sum_{n=0}^{\infty} \prod_{i=1}^{s} \frac{\left(m_{i} !\right)^{\frac{1}{\lambda_{i}}}}{\left(n \alpha k+x_{i}+\alpha-1\right)^{\frac{m_{i}+1}{\lambda_{i}}}} \\
& \leq \prod_{i=1}^{s}\left(\sum_{n=0}^{\infty}\left[\frac{\left(m_{i} !\right)^{\frac{1}{\lambda_{i}}}}{\left(n \alpha k+x_{i}+\alpha-1\right)^{\frac{m_{i}+1}{\lambda_{i}}}}\right]^{\lambda_{i}}\right)^{\frac{1}{\lambda_{i}}} \\
& =\prod_{i=1}^{s}\left|\psi_{\alpha, k}^{\left(m_{i}\right)}\left(x_{i}\right)\right|^{\frac{1}{\lambda_{i}}}
\end{aligned}
$$

which gives the desired result (31).

Remark 4.2. By letting $s=2, \lambda_{1}=p, \lambda_{2}=q, m_{1}=m, m_{2}=n, x_{1}=x$ and $x_{2}=y$ in (31), we get the result

$$
\left|\psi_{\alpha, k}^{\left(\frac{m}{p}+\frac{n}{q}\right)}(x+y)\right| \leq\left|\psi_{\alpha, k}^{(m)}(x)\right|^{\frac{1}{p}}\left|\psi_{\alpha, k}^{(n)}(y)\right|^{\frac{1}{q}}
$$

Furthermore, if $m=n$ in (32), then we obtain

$$
\left|\psi_{\alpha, k}^{(m)}(x+y)\right| \leq\left|\psi_{\alpha, k}^{(m)}(x)\right|^{\frac{1}{p}}\left|\psi_{\alpha, k}^{(m)}(y)\right|^{\frac{1}{q}}
$$

Theorem 4.3. Let $x_{i}>0, u \geq 1$ and $m_{i} \in \mathbb{N}, i=1,2, \ldots, s$. Then the inequality

$$
\left(\sum_{i=1}^{s}\left|\psi_{\alpha, k}^{\left(m_{i}\right)}\left(x_{i}\right)\right|\right)^{\frac{1}{u}} \leq \sum_{i=1}^{s}\left|\psi_{\alpha, k}^{\left(m_{i}\right)}\left(x_{i}\right)\right|^{\frac{1}{u}}
$$

is valid. 
Proof. Using (20) together with (16), we obtain

$$
\begin{aligned}
\left(\sum_{i=1}^{s}\left|\psi_{\alpha, k}^{\left(m_{i}\right)}\left(x_{i}\right)\right|\right)^{\frac{1}{u}} & =\left(\sum_{i=1}^{s} \sum_{n=0}^{\infty} \frac{m_{i} !}{\left(n \alpha k+x_{i}+\alpha-1\right)^{m_{i}+1}}\right)^{\frac{1}{u}} \\
& =\left(\sum_{n=0}^{\infty} \sum_{i=1}^{s}\left[\frac{\left(m_{i} !\right)^{\frac{1}{u}}}{\left(n \alpha k+x_{i}+\alpha-1\right)^{\frac{m_{i}+1}{u}}}\right]^{u}\right)^{\frac{1}{u}} \\
& \leq\left(\sum_{n=0}^{\infty}\left[\sum_{i=1}^{s} \frac{\left(m_{i} !\right)^{\frac{1}{u}}}{\left(n \alpha k+x_{i}+\alpha-1\right)^{\frac{m_{i}+1}{u}}}\right]^{u}\right)^{\frac{1}{u}} \\
& \leq \sum_{i=1}^{s}\left(\sum_{n=0}^{\infty}\left[\frac{\left(m_{i} !\right)^{\frac{1}{u}}}{\left(n \alpha k+x_{i}+\alpha-1\right)^{\frac{m_{i}+1}{u}}}\right]^{\frac{1}{u}}\right. \\
& =\sum_{i=1}^{s}\left|\psi_{\alpha, k}^{\left(m_{i}\right)}\left(x_{i}\right)\right|^{\frac{1}{u}}
\end{aligned}
$$

which gives the result (33).

Remark 4.4. By letting $s=2, m_{1}=m, m_{2}=n, x_{1}=x$ and $x_{2}=y$ in (33), we obtain

$$
\left(\left|\psi_{\alpha, k}^{(m)}(x)\right|+\left|\psi_{\alpha, k}^{(n)}(y)\right|\right)^{\frac{1}{u}} \leq\left|\psi_{\alpha, k}^{(m)}(x)\right|^{\frac{1}{u}}+\left|\psi_{\alpha, k}^{(n)}(y)\right|^{\frac{1}{u}}
$$

\section{Conclusion}

Recently, Sarikaya et al. [10] introduced a new analogue of the Gamma function which they called the conformable Gamma function. Motivated by their results, we have established some inequalities involving the conformable Gamma and Polygamma functions. Apart from providing generalizations of some known results in the literature, the established results could trigger new research directions in the area of study.

\section{REFERENCES}

[1] A. Akkurt, M.E. Yildirim and H. Yildirim, Some Inequalities Involving $(\alpha, k)$-Gamma and $(\alpha, k)-$ Beta Functions for Conformable Fractional Integrals, ResearchGate Article, Available online at: https://www.researchgate.net/publication/309857796.

[2] L. M. B. de Costa Campos, Generalized Calculus with Applications to Matter and Forces, CRC Press, Taylor and Francis Group, New York, 2014.

[3] R. Díaz and E. Pariguan, On hypergeometric functions and Pachhammer k-symbol, Divulgaciones Matemtícas, 15(2)(2007), 179-192.

[4] R. Díaz and C. Teruel, q, k-generalized gamma and beta functions, Journal of Nonlinear Mathematical Physics, 12(2005), 118-134.

[5] F. H. Jackson, On q-definite Integrals, Quarterly Journal of Pure and Applied Mathematics, 41(1910), 193-203.

[6] V. Krasniqi and F. Merovci, Some Completely Monotonic Properties for the ( $p, q)$-Gamma Function, Mathematica Balkanica, New Series, 26(2012), Fasc. 1-2.

[7] T. Mansour and A.S. Shabani, Generalization of some inequalities for the $\left(q_{1}, \ldots, q_{s}\right)$ Gamma function, Le Matematiche, LXVII (2012), Fasc. II, 119-130. 
[8] K. Nantomah. E. Prempeh and S. B. Twum, On a $(p, k)$-analogue of the Gamma function and some associated Inequalities, Moroccan Journal of Pure and Applied Analysis, $2(2)(2016), 79-90$.

[9] P. N. Sadjang, On the $(p, q)$-Gamma and the $(p, q)$-Beta functions, arXiv.org, Available online at: https://arxiv.org/pdf/1506.07394v1.pdf.

[10] M.Z. Sarikaya, A. Akkurt, H. Budak, M.E. Yildirim, and H. Yildirim, On Some Special Functions for Conformable Fractional Integrals, ResearchGate Article, Available online at: https://www.researchgate.net/publication/309760605.

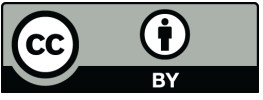

(c) 2016 by the authors; licensee Preprints, Basel, Switzerland. This article is an open access article distributed under the terms and conditions of the Creative Commons Attribution (CC BY) license http://creativecommons.org/licenses/by/4.0/).

Department of Mathematics, Faculty of Mathematical Sciences, University for Development Studies, Navrongo Campus, P. O. Box 24, Navrongo, Ue/R, GHANA; KNANTOMAH@Uds.EDU.GH 